\title{
Silicon-Based Molecular Switch Junctions
}

\author{
Daijiro Nozaki and Gianaurelio Cuniberti $(\bowtie)$ \\ Institute for Material Science and Max Bergmann Center of Biomaterials, Dresden University of Technology, D-01062 Dresden, \\ Germany \\ Received: 4 May 2009 / Revised: 3 June 2009 / Accepted: 3 June 2009 \\ (C)Tsinghua University Press and Springer-Verlag 2009. This article is published with open access at Springerlink.com
}

\begin{abstract}
In contrast to the static operations of conventional semiconductor devices, the dynamic conformational freedom in molecular devices opens up the possibility of using individual molecules as new types of devices such as a molecular conformational switch or for molecular data storage. Bistable molecules - such as those having two stable cis and trans isomeric configurations - could provide, once clamped between two electrodes, a switching phenomenon in the non-equilibrium current response. Here, we model molecular switch junctions formed at silicon contacts and demonstrate the potential of such tunable molecular switches in electrode/molecule/ electrode configurations. Using the non-equilibrium Green function (NEGF) approach implemented with the density-functional-based tight-binding (DFTB) theory, a series of properties such as electron transmissions, current-voltage characteristics in the different isomer conformations, and potential energy surfaces (PESs) as a function of the reaction coordinates along the trans to cis transition were calculated for two azobenzene-based model compounds. Furthermore, in order to investigate the stability of molecular switches under ambient conditions, molecular dynamics (MD) simulations at room temperature were performed and time-dependent fluctuations of the conductance along the MD pathways were calculated. Our numerical results show that the transmission spectra of the cis isomers are more conductive than trans counterparts inside the bias window for both model compounds. The current-voltage characteristics consequently show the same trends. Additionally, calculations of the time-dependent transmission fluctuations along the MD pathways have shown that the transmission in the cis isomers is always significantly larger than that in their trans counterparts, showing that molecular switches can be expected to work as robust molecular switching components.
\end{abstract}

\section{KEYWORDS}

Molecular electronics, molecular switches, charge transport, silicon-molecule interface

\section{Introduction}

Since the successful measurement of current flow through individual molecules in the past few decades, various kinds of molecular devices have been demonstrated by many groups, showing interesting properties such as negative differential resistance [1] and memory effects [2]. The realization of electronic function at the molecular scale, envisioned by Aviram and Ratner in 1974 [3], is gradually finding experimental support due to the development of scanning probe technologies [4,5]. Nevertheless, many challenges still remain. The conductance of a molecule connected between contacts changes

Address correspondence to g.cuniberti@tu-dresden.de 
dramatically depending on many parameters such as the coupling strength between the molecule and the contacts, the electronic structure of the molecule and the contacts, and molecular conformational changes. Therefore, it is highly desirable to understand the relationship between these parameters and transport properties in the design of controllable molecular devices.

In contrast to the static operations of conventional semiconductor devices, the dynamic conformational freedom in molecular devices opens up the possibility of using individual molecules as a new type of device such as a conformational molecular switch or for molecular data storage. The ability to control the conductance at the single molecule scale will have a great impact in nanoscale electronics as well as in other fields such as chemical sensors [6] or medical diagnostics [7]. Photochromic organic molecules having bistable characteristics are attractive materials for the design of molecular switches since a great variety of properties, such as their molecular conformations and their electronic structures, can be reversibly altered from one state to another by external stimulations such as light irradiation and current pulses. Azobenzene, diarylethene, and their derivatives are representative photochromic molecules. Recently, there have been many theoretical and experimental studies of molecular switches [8]. Theoretical studies have explored the use of azobenzene as a molecular switch when connected by $\mathrm{Au}$ electrodes $[9,10]$ or carbon nanotubes (CNTs) [11]. Promising results were obtained, as a significant difference in conductance was seen between the two conformations. Additionally, the study of diarylethene derivatives connected to Au contacts also has shown large changes in conductance between open or closed forms [12].

Several groups have addressed the experimental fabrication of molecular switches consisting of molecules with bistable characteristics sandwiched between contacts. Dulic et al. have created diarylethene derivative-based molecular switch junctions connected between gold contacts by means of the mechanically controlled break junction (MCBJ) technique and measured the current flow through the junction before and after UV irradiation [13]. The current flow through the molecule was suddenly increased when subjected to UV irradiation. Additionally, the current flow did not decay after stopping the irradiation. Although reversible switching has not been observed, conformational switching and the control of current flow through the single molecular device have been realized.

Lörtscher et al. have fabricated molecular junctions where single bipyridyl-dinitro oligophenylene dithiole molecules have been connected between gold contacts with the MCBJ technique [14]. They have demonstrated reversible molecular switching by applying current pulses across the gold contacts. Additionally, Henzl et al. [15, 16] and Choi et al. [17] have reported reversible cis-trans isomerization of a single azobenzene derivative on $\mathrm{Au}(111)$ surfaces induced by the inelastic excitation of vibrational states by an STM tip. Furthermore, Alemani et al. have reported mechanical reversible isomerization of a single azobenzene derivative on the $\mathrm{Au}(111)$ surface induced by an electric field applied from an STM tip not in contact with the molecule [18-20].

In this article, we explore the potential of azobenzene-based molecules as reversibly tunable molecular switches in electrode/molecule/electrode configurations. We have modeled two kinds of molecular switch junctions formed at silicon contacts. In order to improve the molecule-contact coupling, the molecules are covalently attached to Si contacts. We present a detailed analysis of the energetics, potential energy surface (PES) as a function of the reaction coordinate along the trans to cis transition, the stability along molecular dynamics (MD) pathways, and transport properties in the two conformations using the non-equilibrium Green function (NEGF) approach implemented with the density-functionalbased tight-binding (DFTB) method.

This paper is organized as follows. In Section 1 , we present details of the computations used in our study. In Sections 2.1 and 2.2, we show the molecular structures, electronic structures, and PESs of molecular switches in the gas phase and connected between Si contacts. In Section 2.3, we show the transport properties and the stabilities of the molecules between Si contacts. We conclude in Section 3. 


\section{Method}

The relaxation of the molecular structures, total energy calculations, and electronic structure calculations are based on a second-order expansion of the Kohn-Sham total energy in density functional theory with respect to charge density fluctuations [21]. The zeroth order approach is equivalent to a common standard non-selfconsistent tight-binding scheme, while at second order a transparent, parameter-free, and readily calculable expression for generalized Hamiltonian matrix elements can be derived. The resulting DFTB method provides accurate results comparable to the results obtained with ab initio DFT methods or first principles calculations, while requiring fewer computational resources. The method involves modification by selfconsistent redistribution of Mulliken charges. The computations in the DFTB + implementation employed use a minimal basis set, a two-center approximation, neglect of three centers and a crystal field. The atomic pair potentials are computed at a pre-computation stage using a DFT code. The Slater-Koster integrals are stored (as a function of the interatomic distance) in look-up tables which are used during the actual simulation. DFTB + has been extensively validated in the past years by many applications related to, e.g., semiconductor surface reconstructions [22$25]$, reaction of organic molecules [22, 26, 27], and biomolecules [22, 28-30].

Conductance calculations were done via $g D F T B$ code $[31,32]$. The $g D F T B$ code is an extension of the DFTB method to include the NEGF formalism combined with the Landauer formalism. The gDFTB code is based on a tight-binding representation of the wavefunction and a density functional formulation of the Coulomb and exchange correlation potentials. The conductance at low or zero bias (equilibrium) is obtained from electronic transmission probability $T$ as $G=G_{0} T(E, V=0)$, where $G_{0}=2 e^{2} / h$ is the quantum conductance, $e$ is the electron charge, and $h$ is the Plank constant. The electronic transmission probability is related to the retarded/advanced Green function of the molecular region $G^{\mathrm{R} / \mathrm{A}}(E)$ and the self-energies of the electrodes $\sum^{\mathrm{R} / \mathrm{A}}(E)$ by the Fisher and Lee relation [33] $T(E, V)=4 \operatorname{Tr}\left[G^{\mathrm{R}}(E) \operatorname{Im} \cdot \sum_{\mathrm{L}}(E) G^{\mathrm{A}}(E) \operatorname{Im} \sum_{\mathrm{R}}(E)\right]$. We emphasize that when calculating the current $I(V)=(2 e)$ h) $\int d E\left[f_{\mathrm{L}}(E)-f_{\mathrm{R}}(E)\right] T(E, V)$, the full voltage-dependent transmission, $T(E, V)$ is calculated through a selfconsistent Poisson-NEGF cycle in $g D F T B$. Here, $f_{\mathrm{L} / \mathrm{R}}(E)$ are the Fermi functions of the left/right electrodes. The minimal basis set used in $g D F T B$ could be responsible for inaccuracies in the calculation of the current. This may result in problems describing transport mediated via the tails of the wavefunctions. This problem could be solved by using an ab initio code with a richer basis set, but would dramatically increase the computational requirements without adding any qualitatively new results to our treatment.

\section{Results and discussion}

Figure 1(a) shows the two model systems considered in this work. Two different pairs of linkers are attached to the azobenzene molecules. We refer to these two model systems as system A and system B. In each system, the two linkers covalently connecting the molecule to silicon contacts are substituted at the meta- and ortho-positions of the phenyl rings. Large movements of one phenyl ring involved with the cis-trans isomerization are unfavorable for the stable operation of molecular switching since such large movements prevent stable coupling between the molecule and Si contacts. However, via the

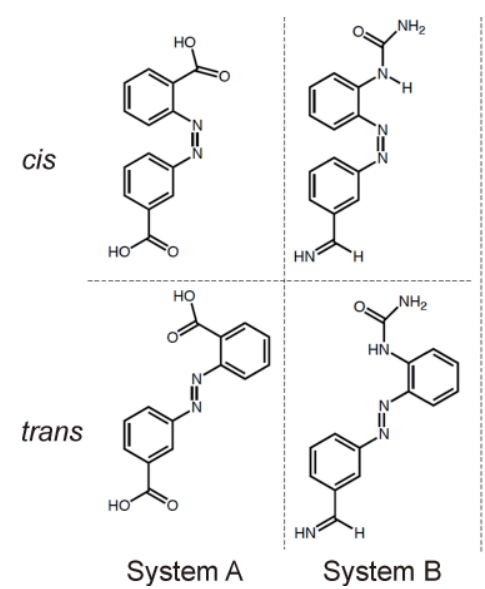

(a)

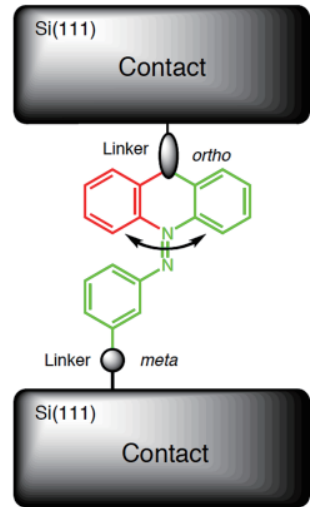

(b)
Figure 1 (a) The two azobenzene derivatives modeled in this work and (b) a schematic illustration of an azobenzene-based molecular switch connected to Si contacts via linkers. The two linkers are substituted at the meta- and ortho- positions of the phenyl rings. These substitution positions reduce the movement of contact atoms during cis-trans isomerization 
linkers at the meta- and ortho-positions, unfavorable displacement at the molecule-contact interface can be reduced as shown in Fig. 1(b). Additionally, the change in molecular length on going from one isomeric state to the other is small (see Table 1) so that the molecules do not tilt on undergoing the cis-trans isomerization. The atoms at the contacts do not move throughout the cis-trans isomerization. Thus, the choice of these positions for the substitutuents is preferable for the stable operation of molecular switching.

Table 1 Molecular length of two molecular switches in gas phase: The molecular lengths are defined by the atomic pairs in parentheses which make bonds with silicon surfaces at top and bottom. These values are obtained after structure optimization by DFTB+ calculations. For the detail see the text.

\begin{tabular}{c|c|c}
\hline & System $\mathrm{A}(\mathrm{O}-\mathrm{O})$ & System $\mathrm{B}(\mathrm{N}-\mathrm{N})$ \\
\hline cis & $10.208 \AA$ & $11.383 \AA$ \\
\hline trans & $11.000 \AA$ & $10.685 \AA$ \\
\hline
\end{tabular}

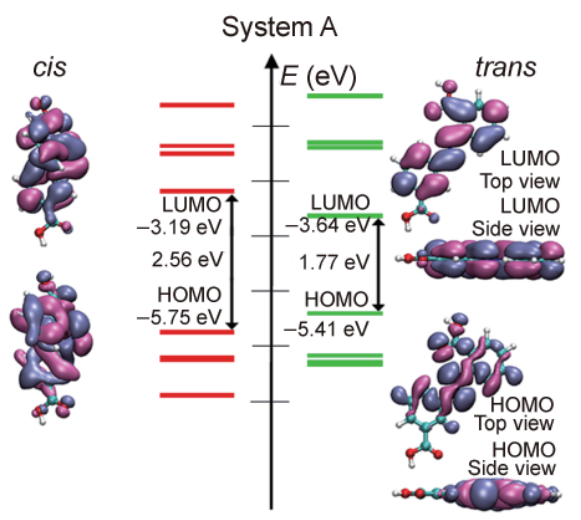

(a)

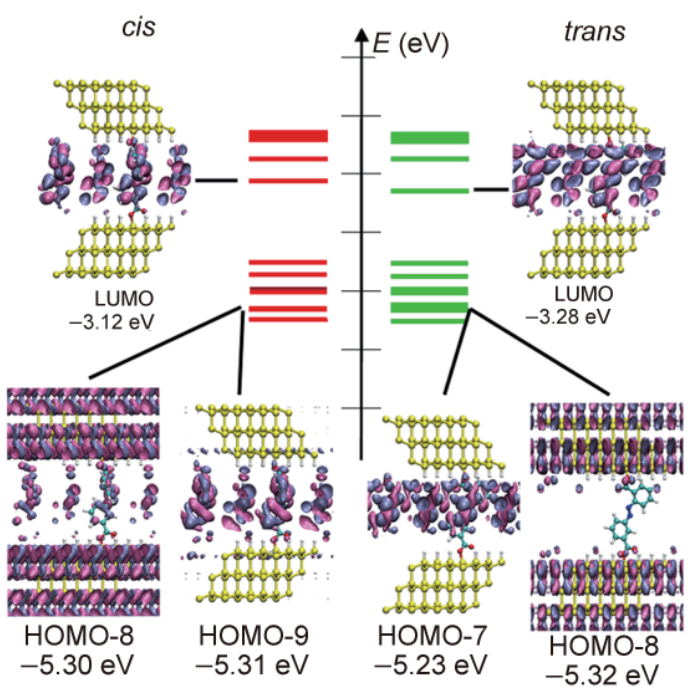

(b)

\subsection{Molecules in the gas phase}

For the determination of the stable configurations of the molecules between the Si contacts, we first performed a geometry optimization for the isolated molecules by means of the conjugate-gradient technique. The geometry optimization was carried out until the absolute value of the interatomic force reduced to less than 10 atomic units. We used the Slater-Koster (SK) parameters developed by Elstner et al. for the C, H, N, O atoms [22] and those of Szuecs et al. for the Si atom [24, 25] as basis sets. Figures 2(a) and 2(c) show the optimized structures, the frontier orbitals, and the molecular energy levels of systems A and B, respectively. The trans isomers are characterized by planar structures while in each cis isomer two phenyl rings are twisted relative to each other by $12.9^{\circ}$ in both systems A and B.

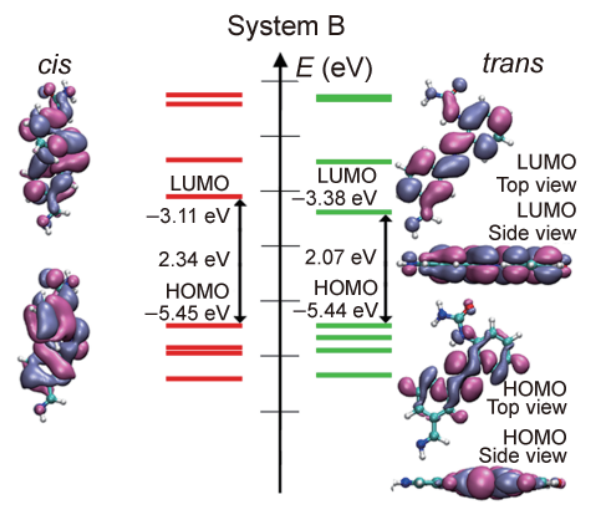

(c)

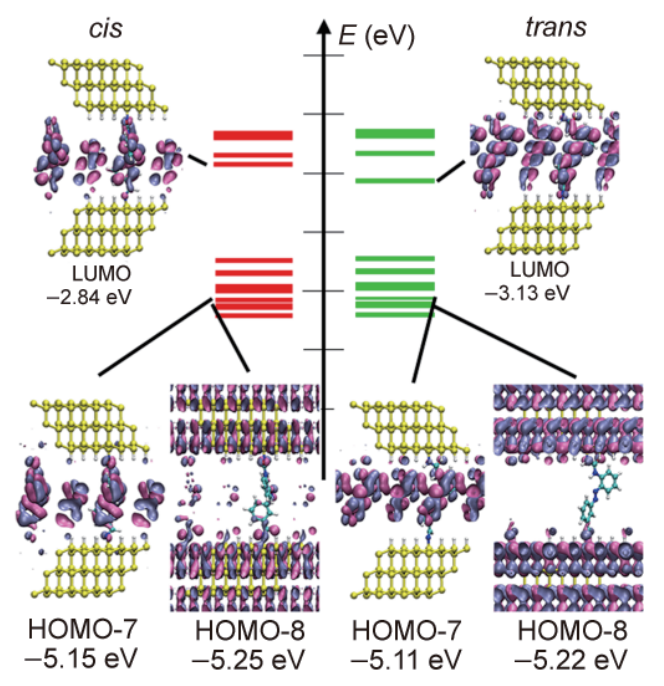

(d)

Figure 2 Energy level diagram and frontier orbitals of system A (a) in the gas phase and (b) between Si contacts. Energy level diagram and frontier orbitals of system $B(c)$ in the gas phase and $(d)$ between Si contacts

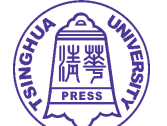


Figure 3(a) shows the PES of total energy as a function of the reaction coordinates along the trans to cis transition for the two model systems in the gas phase. The total energy calculations showed that the trans isomers in systems A and B are energetically more stable than their cis counterparts by $0.193 \mathrm{eV}$ and $0.277 \mathrm{eV}$, respectively. The trans to cis potential energy barriers for systems A and B are $1.060 \mathrm{eV}$ and $1.433 \mathrm{eV}$, respectively. These features are similar to the azobenzene molecule in the gas phase where its trans isomer is experimentally reported to be more stable than the cis counterpart by 0.6 $\mathrm{eV}$ and the potential energy barrier from the trans to the cis counterpart is $1.0 \mathrm{eV}$ [34].

\subsection{Molecules between Si contacts}

Let us consider the model systems connected between Si contacts. Hereafter we refer to these coupled systems as "extended molecules". The

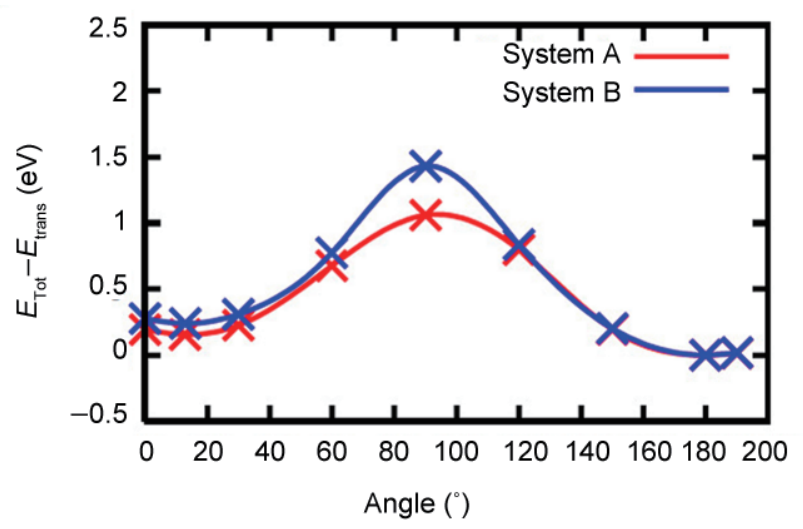

(a)

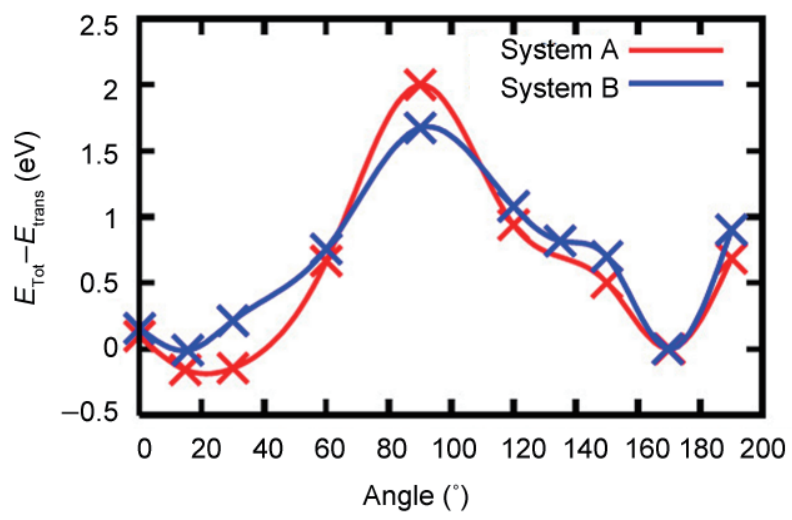

(b)

Figure 3 Potential energy surfaces of systems A and B (a) in the gas phase and (b) between Si contacts. The total energies of both systems are plotted as a function of the dihedral angle between the two bonds connecting nitrogen atoms and phenyl rings. The curves interpolate points, which are indicated by the crosses complexity of the systems leads to a very complicated PES with many metastable states; for simplicity we only consider the vertical arrangements of the molecules with respect to the hydrogen-passivated $\mathrm{Si}(111)$ surface. The structure-optimized molecules in the gas phase are covalently connected to hydrogenpassivated $\mathrm{Si}(111)$ surfaces and relaxed again using DFTB + with periodic boundary conditions. The lateral spacing between azobenzene molecules is set large enough to exclude lateral interactions between them. In each unit cell, the top/bottom silicon contacts are comprised of $54 \mathrm{Si}$ atoms forming three silicon layers and 8 hydrogen atoms covering the $\mathrm{Si}(111)$ surface. In order to include the reconstruction of the silicon atoms on the surfaces, the first two silicon layers of each electrode from the surface are relaxed, while the third layer remains fixed, which is required for the subsequent conductance calculations. The Si-Si bond lengths in the fixed layers are set as $2.35 \AA$, which is the experimental value for bulk $\mathrm{Si}$ crystal. Figure S-1 in the Electronic Supplementary Material (ESM) shows the total energy of the extended molecules as a function of the separation of two Si surfaces. Figure 4 shows the optimized unit cells of two model systems at the separation of Si surfaces where the total energies of trans isomers between $\mathrm{Si}$ contacts are their local minima, which are shown by vertical dotted lines in Fig. S-1 (in the

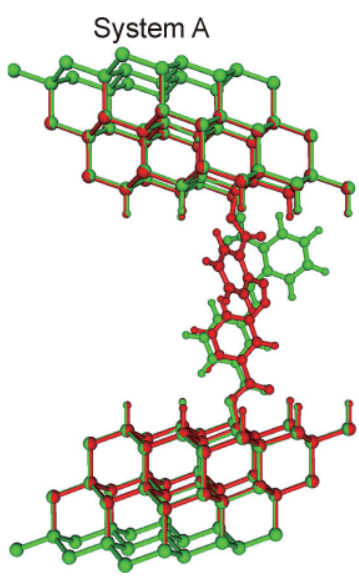

(a)

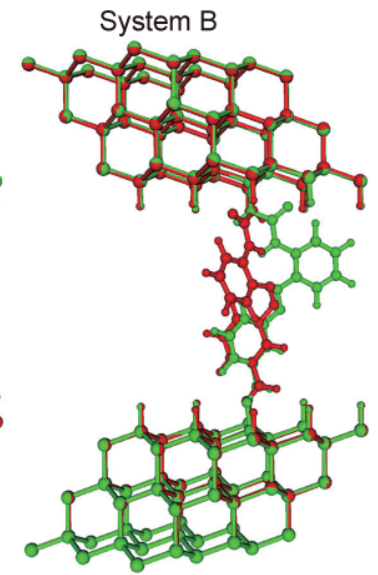

(b)
Figure 4 Optimized structure (unit cell) of the two molecular switch junctions modeled in this work. cis conformations are shown in red and trans conformations in green. For comparison, the two isomer conformations are superimposed. Optimizations are carried out using the conjugated-gradient technique with DFTB + under periodic boundary conditions. The silicon layers at the bottom and top are fixed 
ESM). The PES calculations for extended molecules and subsequent conductance calculations are carried out at these separations.

The PESs of total energies in the extended molecules are shown in Fig. 3(b). The potential energy barriers of extended molecules along the reaction coordinate from trans to cis isomers in systems $\mathrm{A}$ and $\mathrm{B}$ are $2.001 \mathrm{eV}$ and $1.675 \mathrm{eV}$, respectively. The potential energy barriers are increased compared with those in the gas phase (Fig. 3(a)). This is due to the reduction of the conformational degree of freedom of molecules confined between Si contacts.

\subsection{Transport properties}

Once the relaxed geometries have been determined, we first focus on the electronic transmission probabilities of molecular switch junctions for cis and trans isomer conformations at equilibrium. The structureoptimized unit cell of the extended molecules is put between semi-infinite Si bulk layers and then transmission calculations are carried out. Figure 5 shows the electronic transmission probabilities at equilibrium. The Fermi energies are near the edge of the valence band (for the Fermi energies, see Table 2 ). Thus, the conductance in these model systems is driven by hole transport under small bias. Our results show that the cis isomers are more conductive than the trans counterparts around the Fermi energies in both of the model systems. The low conductance between the resonant peaks indicates that the transport can only take place through tunneling. The conductance around the Fermi energy in cis isomers is higher than that for the trans counterparts by a few orders of magnitude. This suggests that switching behavior may be expected at low bias. The low transmissions around the Fermi energies are due to the weak coupling between the molecules and the $\mathrm{Si}$ contacts giving rise to narrow resonant peaks. As shown in the density of states (DOS) profiles of both system A and B in Fig. 6, the narrow resonant peaks due to the HOMO and LUMO of isolated molecules are located at about $-5.0 \mathrm{eV}$ and $-3.0 \mathrm{eV}$, respectively. The Fermi energies lie in the center of gaps between these narrow resonant peaks due to the HOMO and LUMO of isolated molecules. Hence, the DOS on the molecules around the Fermi energies are very low.

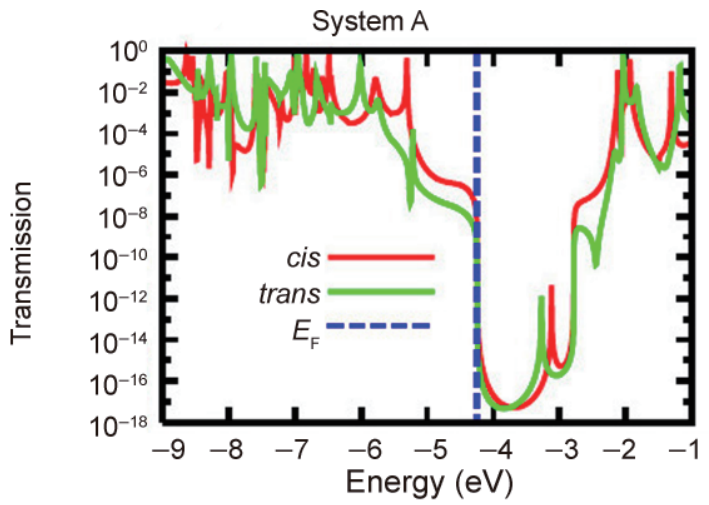

(a)

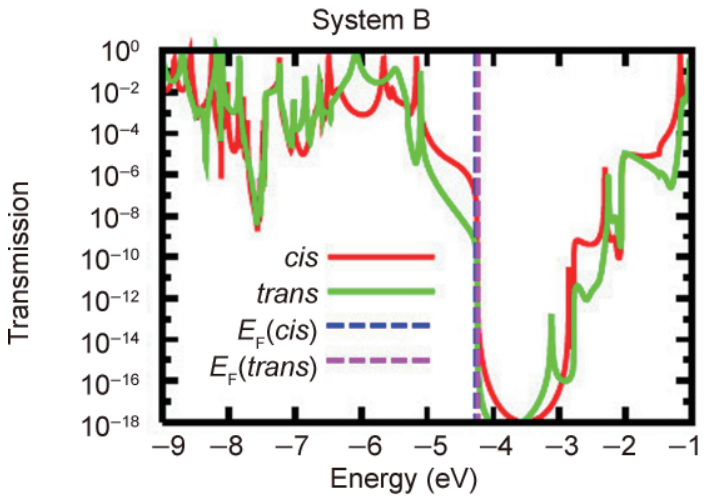

(b)

Figure 5 Electronic transmission probabilities for systems $A$ and $B$ at equilibrium. Transmission spectra for cis conformations are shown in red and for trans conformations in green. Broken lines show the Fermi energies $\left(E_{\mathrm{F}}\right)$. In $(\mathrm{a})$, the Fermi energies for the two isomers are same. Thus, only one broken line is shown. For details of the Fermi energies, see Table 2

Table 2. Fermi energies of two molecular switching junctions.

\begin{tabular}{c|c|c}
\hline & System A & System B \\
\hline cis & $-4.259 \mathrm{eV}$ & $-4.255 \mathrm{eV}$ \\
\hline trans & $-4.259 \mathrm{eV}$ & $-4.253 \mathrm{eV}$ \\
\hline
\end{tabular}

Previous theoretical studies of azobenzene derivatives connected between Au $[9,10]$ or CNT electrodes [11] have reported that cis isomers are less conductive than their trans counterparts, since the $\pi$-conjugation in the trans isomers along the molecular framework is broken by the torsion involved with cis-trans isomerizations. However, the same discussion does not hold true in our case since the HOMO of the trans isomers does not consist of $p_{z}$ orbitals perpendicular to the molecular framework, but non-binding orbitals mainly localized around nitrogen atoms [35, 36], as shown in Figs. 2(a) and 2(c). The eigenstates consisting of $p_{z}$ orbitals of the

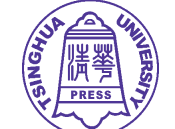


trans isomers lie in the HOMO-3 levels. Thus, these states do not contribute to the transport at low bias. This counter-intuitive result $(I($ cis $)>I($ trans $))$ can also be understood by analysis of the resulting mixture states of the extended molecular systems as follows.

Figure 5 shows that the transmission curves for cis isomers near the Fermi energies are higher than those of trans counterparts, implying there will be a higher current through the cis isomers than the trans counterparts at low bias. The difference between the transmission curves of the cis and trans isomers near the Fermi energies comes from the difference between the height of the resonant peaks of the cis and trans isomers around $-5.0 \mathrm{eV}$. The height of the transmission resonant peaks in the two different isomer conformations can be interpreted from the molecular orbitals of the extended molecules. The HOMOs of the cis isomers interact with surface states of the Si contacts and their orbitals hybridize. The resulting mixed states, corresponding to the HOMO-8 of system A in Fig. 2(b) and of system B in Fig. 2(d), have a non-negligible orbital distribution bridging across the Si states. These states can contribute to transport as shown in the resonance peaks (in red) of system A at $-5.31 \mathrm{eV}$ in Fig. 5(a), and of system B at $5.17 \mathrm{eV}$ in Fig. 5(b).

On the other hand, the HOMOs of the trans isomers have neither orbital amplitude around the linkers nor interact strongly with the surface states of the Si contacts. This leads to less orbital hybridization as can be seen for HOMO-7 of Figs. 2(b) and 2(d). These states do not contribute to transport as shown by the sharper and smaller resonant peaks (in green) for system A at $-5.22 \mathrm{eV}$ in Fig. 5(a) and for system $\mathrm{B}$ at $-5.10 \mathrm{eV}$ in Fig. 5(b). Despite the delocalized molecular orbitals along the molecular framework, the LUMOs of both the trans and cis isomers in both model systems do not interact with the surface state of the Si surface. This is because the energetic difference between the LUMOs of both cis and trans isomers and the conduction state of the Si surface (the conduction band is above $-2.70 \mathrm{eV}$ and the valence band is below $-4.51 \mathrm{eV}$ ) is large. Additionally, in both model systems, there are no state available in the silicon contacts at the energy around the LUMOs of either the trans and cis isomers. Thus, these

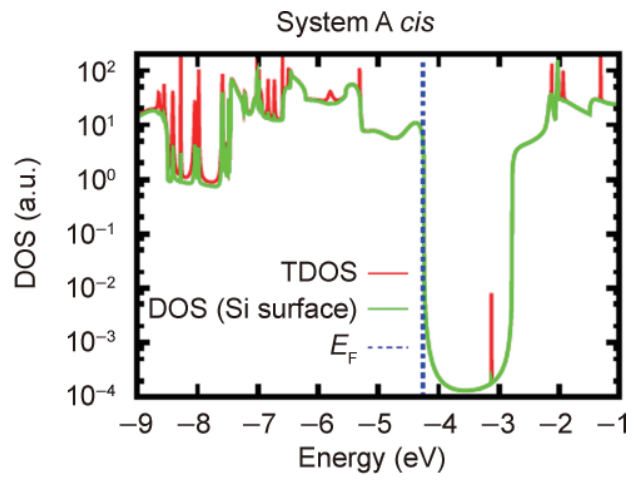

(a)

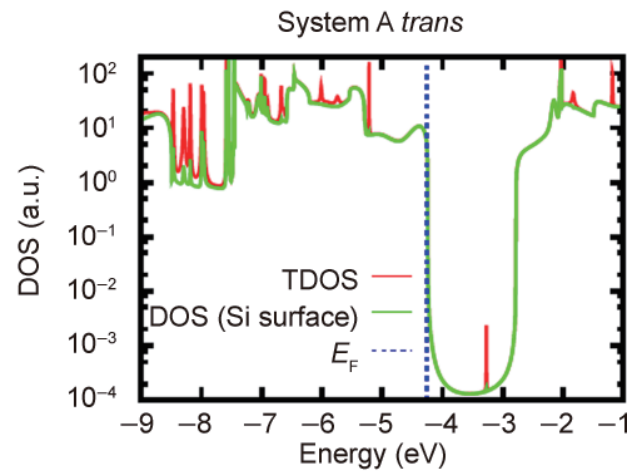

(b)

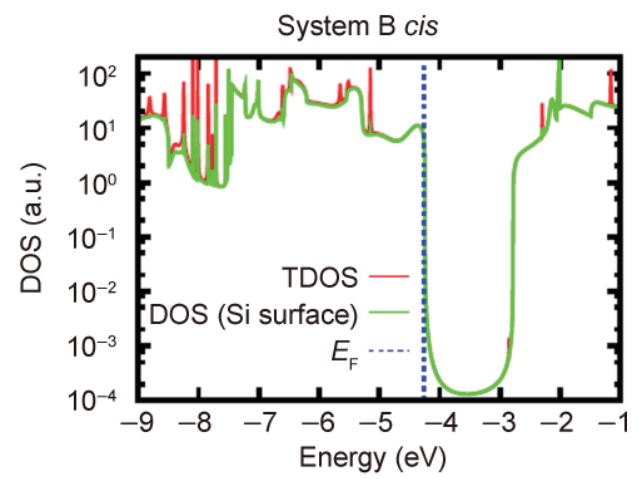

(c)

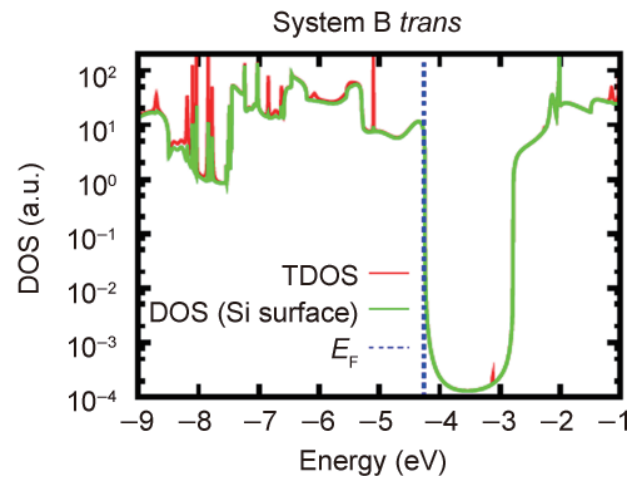

(d)

Figure 6 Total densities of states (TDOS) for systems A and B at equilibrium. TDOS are shown in red curves. The contributions from $\mathrm{Si}$ surfaces to TDOS are shown in green curves. Broken lines show the Fermi energies $\left(E_{\mathrm{F}}\right)$ 
states lead to much lower peaks around $-3.0 \mathrm{eV}$ in transmission spectra as shown in Fig. 5.

We have to pay attention not only to the $\pi$-conjugation but also to the interaction between the molecules and the contacts. These results support the conclusion that the electronic structure of both molecule and contact are crucial in determining the transport properties at low bias.

In order to confirm the possible switching behavior already suggested by the analysis of transmission probabilities at equilibrium, we have calculated the current-voltage $(I-V)$ characteristics for the two different isomer conformations of the two model systems. The $I-V$ characteristics for cis and trans conformations for the two model systems are shown in Fig. 7. The charge transport in trans isomers is strongly suppressed compared to that in the cis counterparts for both of the two model systems. If the bias voltage is less than $1.0 \mathrm{~V}$, the resonant peaks do not enter the Fermi bias window so that the $I-V$ curves are smooth within the bias window. Therefore, we can expect constant on-off ratios as a response to the change or fluctuation of bias voltage, which are preferable for the robust control of the molecular switching. The bottom panels in Figs. 7(a) and 7(b) show the on-off ratios (the ratio of current in the cis isomer to that in the trans counterpart). The onoff ratio reaches as high as 647 for system B at $0.2 \mathrm{~V}$ (bottom panel of Fig. 7(b)). Although the on-off ratios decrease with higher bias voltage, all the molecular switches recorded on-off ratios of over 10 within $1.0 \mathrm{~V}$. Higher on-off ratios for lower bias voltage are advantageous both for power saving and for the suppression of unexpected breakdown triggered at higher bias voltage.

\subsection{Time dependent fluctuation of conductance}

For the reliable operation of molecular switching one needs to investigate the stability of the model systems between Si contacts and the fluctuation of the current through them under ambient conditions. For this purpose, we have performed MD simulations of the model systems between Si contacts and calculated the conductance of the two different isomer conformations along the MD pathways. The calculation setting was as follows. We used the Verlet algorithm for the MD simulation in DFTB + with periodic boundary conditions. We used the same SK parameters used for the structure optimization. The MD duration is set for 10 ps, i.e., 10,000 steps at intervals of $1.0 \mathrm{fs}$. The nuclear and electronic temperatures are set as $300 \mathrm{~K}$. After performing MD simulations (animations of the results of the MD simulations are shown in the ESM), the coordinates in every 100 steps are extracted from MD trajectories. Finally, we repeated conductance

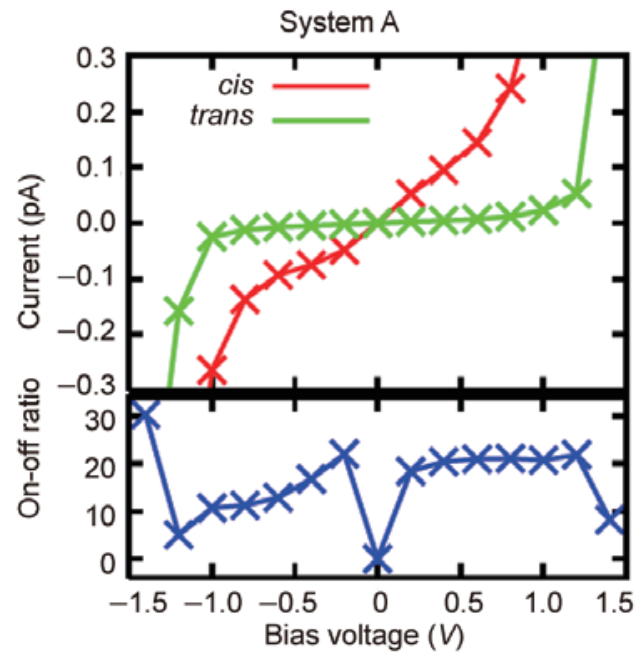

(a)

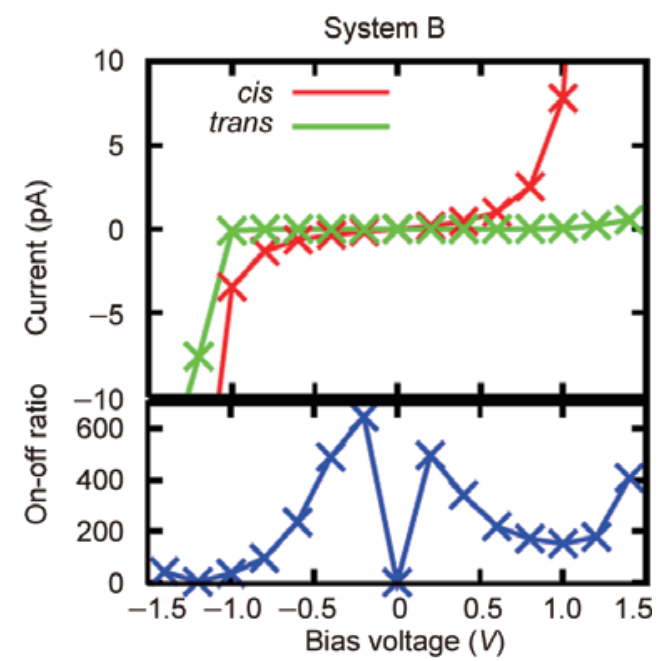

(b)

Figure $7 \quad I-V$ characteristics of molecular switch junctions in the two different conformations; the bottom panels show the on-off ratios (the ratio of current between cis and trans isomers). In all cases, the cis conformations (red) are more conductive than their trans counterparts (green) 
calculations for the collected 100 coordinates using the $g D F T B$ code, both with and without applied bias voltage. We then analyzed the fluctuation of the transmission probabilities and $I-V$ curves along the MD pathways.

The transmission probabilities along the MD pathways are plotted in Fig. S-2 (in the ESM). For each isomer, 100 transmission spectra are plotted. We can see that the main features of the transmission spectra in Fig. 5 remain unchanged. Hence, we can expect that the fluctuation of current flow under ambient conditions is small. The dispersion of the current along the MD pathways as a function of bias voltage is plotted in Fig. 8. The dispersion of the current in the two isomers is distinguishable, with the cis isomers always being more conductive than the trans counterparts. The currents in the two isomers as a function of time under bias $(0.2 \mathrm{~V})$ shown in Fig. 9 also support this conclusion. The onoff ratio is over 15 (the minimum value is 15.5 at 4.7 ps in Fig. 9(b)) for all model systems. Therefore, the molecular switch junctions modeled on this study may be expected to work as switching components under ambient conditions.

Finally, we would like to comment on the possible experimental realization of molecular conformational switching devices consisting of azobenzene derivatives and Si contacts. The attachment of aromatic compounds to the Si surface should be possible using the methodology developed by Murata et al. [37-39]. The realization of two terminal Si/ molecule/Si devices by covering the functionalized Si surfaces from the top with a Si contact might present a formidable challenge due to the intrinsic asymmetry in molecular junctions. However, first attempts at such a difficult task have already proved

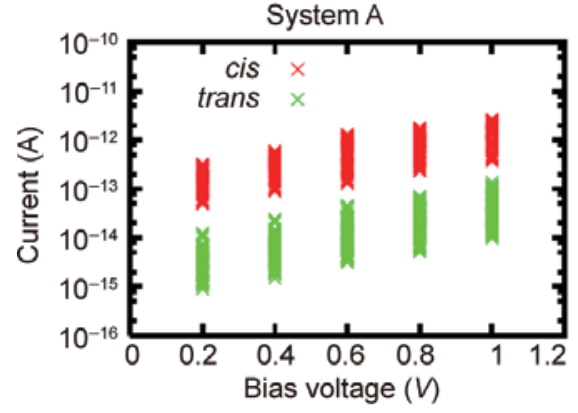

(a)

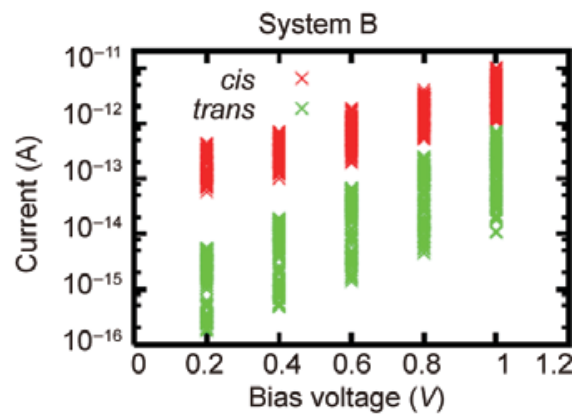

(b)

Figure 8 Dispersion of currents along MD pathways as a function of bias voltage. For each bias voltage 100 values of the current in the cis-isomers along the MD pathways are plotted in red crosses and those for the trans-isomers are plotted in green crosses

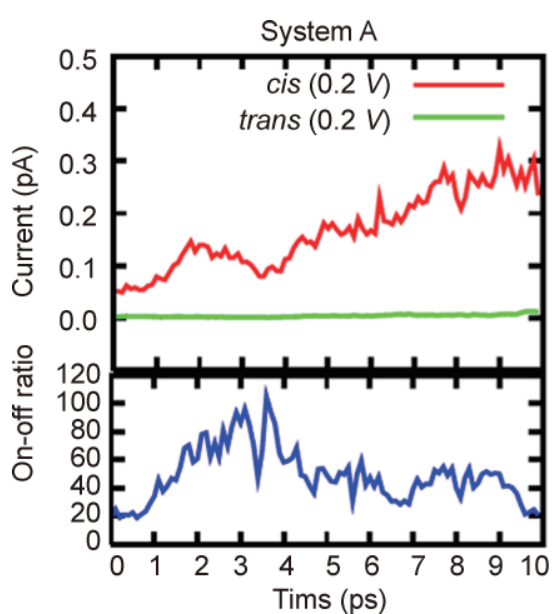

(a)

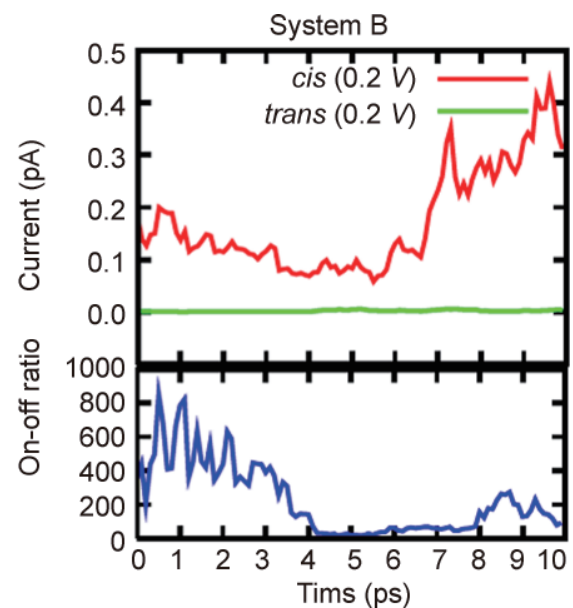

(b)

Figure 9 Time-dependent fluctuation of current (top) and on-off ratio (bottom) along MD pathways at fixed bias voltage in the molecular switch junctions 
successful $[40,41]$. For further understanding and control of the molecular switching, systematic studies on the effect of contacts $[42,43]$, internal scattering effects [44], surface roughness, and impurities [45] will be required. The spacing between the two Si surfaces will also be an important factor since it will influence the PES, molecular and electronic structure, and the resulting conductivity of the molecule embedded between Si contacts. Concerning the design of azobenzene derivatives, further investigation is required to determine which linkers and functional groups lower the PES barrier for cistrans isomerization. One possibility to achieve this is to increase the flexibility of the molecule by using long linkers which should not, however, increase the fluctuation of current due to the flexibility of the molecules.

\section{Conclusions}

We have modeled two molecular switch junctions consisting of azobenzene derivatives and silicon contacts and explored their potential as molecular switches by analysis of the energetics, PES, and transport properties in the two different isomer conformations: cis and trans. Modeling of the molecular switches demonstrated that the attachment of linkers at the meta- and ortho- positions could reduce the steric repulsion between the molecules and silicon contacts during cis-trans isomerization to a value which is reasonable for the robust operation of molecular switching. Our numerical results show that cis isomers are more conductive than the trans counterparts inside the bias window for both model systems. $I-V$ characteristics also show the same trend. Although the $\pi$-conjugation through the molecular framework is reduced due to the torsion of the two phenyl rings, the cis isomers interact much more strongly with the surface states of the silicon contacts than their trans counterparts, resulting in larger conductivities. Additionally, the calculation of currents through the two model systems along the MD pathways under ambient conditions revealed that the currents in the cis isomers are always significantly larger than in the trans counterparts leading to high on-off ratios. Importantly, the dispersion of the current as a function of time is small enough to distinguish the two different isomer conformations. Thus, azobenzene derivatives between Si contacts are promising for applications as robust molecular switching components or as single molecular data storage devices.

\section{Acknowledgements}

This work has been partially funded by the Volkswagen Foundation by the Deutsche Forshuhgsgemeinschaft (DFG) under Contracts No. CU 44/5-2, CU 44/8-1, and CU 44/3-3, and by the WCU (World Class University) program through the Korea Science and Engineering Foundation funded by the Ministry of Education, Science and Technology (Project No. R31-2008-000-10100-0). We acknowledge the Center for Information Services and High Performance Computing (ZIH) at the Dresden University of Technology for computational resources. We thank Rafael Gutiérrez, Haldun Sevinçli, Florian Pump and Cormac Toher for fruitful discussions.

Electronic Supplementary Material: Supplementary material is available in the online version of this article at http://dx.doi.org/10.1007/s12274-0099067-y and is accessible free of charge.

\section{References}

[1] Chen, J.; Reed, M. A.; Rawlett, A. M.; Tour, J. M. Large on-off ratios and negative differential resistance in a molecular electronic device. Science 1999, 286, 1550-1552.

[2] Cuniberti, G.; Fagas, G.; Richter, K. Introducing Molecular Electronics; Springer-Verlag Berlin, Heidelberg, 2005.

[3] Aviram, A.; Ratner, M. A. Molecular rectifiers. Chem. Phys. Lett. 1974, 29, 277.

[4] Fernández-Torrente, I.; Franke, K. J.; Pascual, J. I. Vibrational Kondo effect in pure organic charge-transfer assemblies. Phys. Rev. Lett. 2008, 101, 217203.

[5] Schulze, G.; Franke, K. J.; Gagliardi, A.; Romano, G.; Lin, C. S.; Rosa, A. L.; Niehaus, T. A.; Frauenheim, T.; Di Carlo, A.; Pecchia, A.; Pascual, J. I. Resonant electron heating and molecular phonon cooling in single C-60 junctions. Phys. Rev. Lett. 2008, 100, 136801. 
[6] Cui, Y.; Wei, Q.; Park, H.; Lieber, C. M. Nanowire nanosensors for highly sensitive and selective detection of biological and chemical species. Science 2001, 293, 1289-1292.

[7] Patolsky, F.; Zheng, G.; Hayden, O.; Lakadamyali, M.; Zhuang, X.; Lieber, C. M. Electrical detection of single viruses. Proc. Natl. Acad. Sci. USA 2004, 101, 1401714022

[8] Feringa, B. L. Molecular Switches; Wiley-VCH: Weinheim, 2001.

[9] Zhang, C.; Du, M. -H.; Cheng, H. -P.; Zhang, X. -G.; Roitberg, A. E.; Krause, J. L. Coherent electron transport through an azobenzene molecule: A light-driven molecular switch. Phys. Rev. Lett. 2004, 92, 158301/1-4.

[10] Zhang, C.; He, Y.; Cheng, H. -P.; Xue, Y.; Ratner, M. A.; Zhang, X. -G.; Krstic, P. Current-voltage characteristics through a single light-sensitive molecule. Phys. Rev. B 2006, 73, 125445

[11] del Valle, M.; Gutierrez, R.; Tejedor, C.; Cuniberti, G. Tuning the conductance of a molecular switch. Nat. Nanotechnol. 2007, 2, 176-179.

[12] Staykov, A.; Nozaki, D.; Yoshizawa, K. Photoswitching of conductivity through a diarylperfluorocyclopentene nanowire. J. Phys. Chem. C 2007, 111, 3517-3521.

[13] Dulic, D.; van der Molen, S. J.; Kudernac, T.; Jonkman, H. T.; de Jong, J. J. D.; Bowden, T. N.; van Esch, J.; Feringa, B. L.; van Wees, B. One-way optoelectronic switching of photochromic molecules on gold. J. Phys. Rev. Lett. 2003, 91, 207402/1-4

[14] Lörtscher, E.; Ciszek, J. W.; Tour, J.; Riel, H. Reversible and controllable switching of a single-molecule junction. Small 2006, 2, 973-977.

[15] Henzl, J.; Mehlhorn, M.; Gawronski, H.; Rieder, K. -H.; Morgenstern, K. Reversible cis-trans isomerization of a single azobenzene molecule. Angew. Chem. Int. Ed. 2006, 45, 603-606.

[16] Henzl, J.; Mehlhorn, M.; Morgenstern, K. Aminonitro-azobenzene dimers as a prototype for a molecular-level machine. Nanotechnology 2007, 18, 495502-1-6.

[17] Choi, B. -Y.; Kahng, S. -J.; Kim, S.; Kim, H.; Kim, H. W.; Song, Y. J.; Ihm, J.; Kuk, Y. Conformational molecular switch of the azobenzene molecule: A scanning tunneling microscopy study. Phys. Rev. Lett. 2006, 96, 156106.

[18] Alemani, M.; Peters, M. V.; Hecht, S.; Rieder, K. -H.; Moresco, F.; Grill, L. Electric field-induced isomerization of azobenzene by STM. J. Am. Chem. Soc. 2006, 128,
14446-14447.

[19] Alemani, M.; Selvanathan, S.; Ample, F.; Peters, M. V.; Rieder, K. -H.; Moresco, F.; Joachim, C.; Hecht, S.; Grill, $\mathrm{L}$. Adsorption and switching properties of azobenzene derivatives on different noble metal surfaces: Au(111), $\mathrm{Cu}(111)$, and $\mathrm{Au}(100)$. J. Phys. Chem. C 2008, 112, 10509-10514.

[20] Dri, C.; Peters, M. V.; Schwarz, J.; Hecht, S.; Grill, L. Spatial periodicity in molecular switching. Nat. Nanotechnol. 2008, 3, 649-653.

[21] Frauenheim, T.; Seifert, G.; Elstner, M.; Hajnal, Z.; Jungnickel, G.; Porezag, G.; Suhai, S.; Scholz, R. A selfconsistent charge density-functional based tight-binding method for predictive materials simulations in physics, chemistry and biology. Phys. Status Solidi B 2000, 217, 41-62.

[22] Elstner, M.; Porezag, G.; Jonkman, H. T.; Elsner, J.; Haugk, M.; Frauenheim, T.; Suhai, S.; Seifert, G. Selfconsistent-charge density-functional tight-binding method for simulations of complex materials properties. Phys. Rev. B 1998, 58, 7260-7268.

[23] Haugk, M.; Elsner, J.; Frauenheim, T. A density-functional based tight-binding approach to GaAs surface reconstructions. J. Phys.: Cond. Matt. 1997, 9, 7305-7315.

[24] Szucs, B.; Hajnal, Z.; Frauenheim, T.; González, C.; Ortega, J.; Pérez, R.; Flores, F. Chalcogen passivation of GaAs(100) surfaces: Theoretical study. Appl. Surf. Sci. 2003, 212, 861-865.

[25] Szucs, B.; Hajnal, Z.; Scholz, R.; Sanna, S.; Frauenheim, T. Theoretical study of the adsorption of a PTCDA monolayer on S-passivated GaAs(100). Appl. Surf. Sci. 2004, 234, 173-177.

[26] Krüger, T.; Elstner, M.; Schiffels, P.; Frauenheim, T. Validation of the density-functional based tight-binding approximation method for the calculation of reaction energies and other data. J. Chem. Phys. 2005, 122, 114110.

[27] Niehaus, T. A.; Elstner, M.; Frauenheim, T.; Suhai, S. Application of an approximate density-functional method to sulfur containing compounds. J. Mol. Struct. (THEOCHEM) 2001, 541, 185-194.

[28] Elstner, M.; Frauenheim, T.; Kaxiras, E.; Seifert, G.; Suhai, S. A self-consistent charge density-functional based tight-binding scheme for large biomolecules. Phys. Status Solidi B 2000, 217, 357-376.

[29] Elstner, M.; Hobza, P.; Frauenheim, T.; Suhai, S.; Kaxiras, E. Hydrogen bonding and stacking interactions of nucleic 
acid base pairs: A density-functional-theory based treatment. J. Chem. Phys. 2001, 114, 5149-5155.

[30] Elstner, M.; Jalkanen, K. J.; Knapp-Mohammady, M.; Frauenheim, T.; Suhai, S. Energetics and structure of glycine and alanine based model peptides: Approximate SCC-DFTB, AM1 and PM3 methods in comparison with DFT, HF and MP2 calculations. Chem. Phys. 2001, 263, 203-219.

[31] Di Carlo, A.; Pecchia, A.; Latessa, L.; Frauenheim, T.; Seifert, G. Lect. Notes Phys. 2005, 680, 153.

[32] Pecchia, A.; Di Carlo, A. Atomistic theory of transport in organic and inorganic nanostructures. Rep. Prog. Phys. 2004, 67, 1497-1561.

[33] Fisher, D. S.; Lee, P. A. Relation between conductivity and transmission matrix. Phys. Rev. B 1981, 23, 6851-6854.

[34] Brown, E. V.; Grunneman, G. R. J. Am. Chem. Soc. 1975, $97,621$.

[35] Sauer, P.; Allen, R. E. Multiple steps and multiple excitations in photoisomerization of azobenzene. Chem. Phys. Lett. 2008, 450, 192-195.

[36] Füchsel, G.; Klamroth, T.; Dokic, J.; Saalfrank, P. On the electronic structure of neutral and ionic azobenzenes and their possible role as surface mounted molecular switches. J. Phys. Chem. B 2006, 110, 16337-16345.

[37] Murata, H.; Itabashi, A.; Arai, T.; Tomitori, M. In Electronic Structure and Processes of Molecular-Based Interfaces: In
Relation to Organic and Molecular Devices (ESPMI-06), Nagoya, Japan, 2006, p. 55.

[38] Murata, H.; Itabashi, A. In MRS Fall Meeting, Boston, USA, 2004

[39] Itabashi, A.; Arai, T.; Tomitori, M.; Murata, H. In The Third International Conference on Molecular Electronics and Bioelectronics (M\&BE3), Tokyo, Japan, 2005.

[40] Eberl, K.; Schmidt, O. G. Nanotechnology_Thin solid films roll up into nanotubes. Nature 2001, 410, 168-168.

[41] Deneke, C.; Schumann, J.; Engelhard, R.; Thomas, J.; Sigle, W.; Zschieschang, U.; Klauk, H.; Chuvilin, A.; Schmidt, O. G. Fabrication of radial superlattices based on different hybrid materials. Phys. Status Solidi C 2008, 5, 2704-2708.

[42] Fagas, G.; Cuniberti, G.; Richter, K. Electron transport in nanotube-molecular-wire hybrids. Phys. Rev. B 2001, 63, 045416/1-4.

[43] Cuniberti, G.; Grossmann, F.; Gutiérrez, R. The role of contacts in molecular electronics. Adv. Solid State Phys. 2002, 42, 133-149.

[44] Nozaki, D.; Girard, Y.; Yoshizawa, K. Theoretical study of long-range electron transport in molecular junctions. J. Phys. Chem. C 2008, 112, 17408-17415.

[45] Ciacchi, L. C.; Payne, M. C. First-principles moleculardynamics study of native oxide growth on Si(001). Phys. Rev. Lett. 2005, 95, 196101. 